\title{
Kinetic Theory for Some Reactions Proceeding on a Polymer Chain
}

\author{
Masahiko Sisido \\ Department of Polymer Chemistry, Kyoto University, Kyoto, Japan.
}

(Received July 20, 1971)

\begin{abstract}
Kinetic theories were developed for the reactions on a polymer chain carrying both substrates and catalytic groups randomly. The reaction courses were formulated (1) considering the distribution of the substrates or the catalytic groups along the chain to be time-averaged or (2) considering the distribution explicitly, introducing the probability of finding a catalytic group on each residue, which is determined independently from other residues or (3) introducing the probability that depends upon the states of the neighboring residues. These theories were successfully applied to the experimental work reported by Morawetz, et al., and subsequently discussed.

KEY WORDS Kinetic Theory / Polymer Reaction / Intramolecular Reaction / Catalytic Group Distribution / Markov Process / Matrix Method /
\end{abstract}

Various reactions proceeding on a polymer chain have been studied by some workers. ${ }^{1-4}$ Most of the reaction processes were accounted for by the neighboring group effect on the polymer chain. However, it should be mentioned that the effects of the catalytic groups located at distant positions from a given reactive group (substrate) along the chain should not be ignored. These "remote group effects" have not been considered probably because there has been no kinetic theory concerning this effect and no information on the parameters to solve the problem. Recently, Morawetz, et al.,$^{5-6}$ also noted this problem and analyzed their experimental results by a computer simulation. However, their method consists of the Monte Carlo estimation of the distribution of catalytic groups on a polymer chain and the analytical equation of the problem has not yet been obtained. In this paper, kinetic theories were developed for the reactions on a polymer chain carrying both catalytic groups and substrates on it. The substrate is assumed to give a non-reactive product by the action of the catalytic groups attached to the same chain. In such a case, the following special circumstances must be considered. Firstly, the reaction can be treated as unimolecular reaction, since the catalytic groups reacting with a given substrate are limited to those attached on the same chain. Secondly, if the positions of substrates and catalytic groups are fixed or nearly immobilized during the course of the reaction, the difference in the distribution of catalytic groups around each substrate causes the difference in the reaction rate for each substrate. The reaction begins with the reactive substrates and transfers to the less reactive ones. Therefore, the firstorder plot of the reaction will not give a straight line. In these cases, the distribution of the catalytic groups on a polymer chain should be taken into consideration for the kinetic treatment. According to the mode of the distribution, the following three cases are distinguished.

(1) The distribution of catalytic groups is time-averaged over all polymer chains in a system, so, the kinetics can be treated without considering the distribution.

(2) The distribution is taken into account introducing the probability for a given residue (monomer unit) to carry a catalytic group that is determined independently from the states of other residues.

(3) The above probability depends on whether or not their neighboring residues carry the catalytic group.

\section{FORMULATIONS}

Treatment 1; Kinetics without Considering the Distribution

Here, the kinetics of polymer reactions is de- 
veloped, in which the substrates and/or the catalytic groups are, for example, adsorbed on a chain being in equilibration with the free substrates and/or free catalytic groups, and the equilibrium is attained very rapidly compared with the reaction rate. In these cases, the probability for a residue to carry a catalytic group, can be regarded as equivalent for all the polymer chains. Let us define $[R]$ as the total substrate concentration attached on polymer chains, $\left[\mathrm{R}_{I}\right]$ as the concentration of substrate attached on the $I$-th residue of the chains and $N$ as the degree of polymerization (the number of the residues) of the chain.

$$
[\mathrm{R}]=\sum_{I=1}^{N}\left[\mathrm{R}_{I}\right]
$$

Further, $k_{I J}$ is defined as the first-order rate constant of the reaction between a substrate on the $I$-th residue and a catalytic group on the $J$-th residue and also $p_{J}$ as a probability for $J$-th residue to carry a catalytic group. Using these quantities, the reaction rate of substrates on the $I$-th residues is represented as

$$
-\mathrm{d}\left[\mathbf{R}_{I}\right] / \mathrm{d} t=\sum_{J=1}^{N} k_{I J} p_{J}\left[\mathbf{R}_{I}\right]
$$

The condition presented in this section allows $p_{J}$ to be written in the averaged form for all the polymer chains

$$
p_{J}=\left[\mathrm{C}_{J}\right] /[\mathrm{P}]
$$

where $\left[\mathrm{C}_{J}\right]$ is the concentration of catalytic groups on the $J$-th residues and [P] means the concentration of polymer chains. $[\mathrm{P}]$ is equal to the concentration of the end groups of the chain, because throughout this paper the effect of the molecular weight distribution of the chains is not considered. The over-all rate can be formulated using the above relations.

$$
\begin{aligned}
\text { rate }=-\mathrm{d}[\mathrm{R}] / \mathrm{d} t & =\sum_{I} \sum_{J} k_{I J} p_{J}\left[\mathrm{R}_{I}\right] \\
& =(1 /[\mathrm{P}]) \sum_{I, J} k_{I J}\left[\mathrm{R}_{I}\right]\left[\mathrm{C}_{J}\right]
\end{aligned}
$$

When the concentrations of substrates and catalytic groups on polymer chains are regarded as constant, the reaction proceeds at a constant rate represented by eq 4 . This situation will be realized when the substrates adsorbed on each residue is in equilibration with the excess of free substrates. In this case, if $\left[\mathrm{R}_{I}\right]$ 's are replaced by equilibrium concentrations, eq 4 will become the sum of the Michaelis-Menten type $^{7}$ equations for each residue. On the other hand, when substrates are bound tightly on the chains and catalytic groups are in an adsorption equilibrium, the concentration of the substrates on $I$-th residue decreases as the reaction proceeds. In this case, the rate of the decrease of $\left[\mathrm{R}_{I}\right]$ must be considered.

$$
-\mathrm{d}\left[\mathrm{R}_{I}\right] / \mathrm{d} t=\left[\mathrm{R}_{I}\right]\left\{(1 /[\mathrm{P}]) \sum_{J} k_{I J}\left[\mathrm{C}_{J}\right]\right\}
$$

The quantity in parentheses, \{\} , is constant and can be replaced by an apparent first-order rate constant $K_{I}$.

$$
K_{I}=(1 /[\mathrm{P}]) \sum_{J} k_{I J}\left[\mathrm{C}_{J}\right]
$$

Using eq 5 and 6 , the over-all rate at time $t$, is obtained

$$
\text { rate }=\sum_{I}\left[\mathrm{R}_{I}\right]_{0} K_{I} \exp \left(-K_{I} t\right)
$$

where subscript 0 means the initial value for the quantity. The fraction of unreacted substrates $y$ is given as

$$
\begin{aligned}
y=[\mathrm{R}] /[\mathrm{R}]_{0} & =\left\{\sum_{I}\left[\mathrm{R}_{I}\right]_{0} \exp \left(K_{I} t\right)\right\} / \sum_{I^{\prime}}\left[\mathrm{R}_{I^{\prime}}\right]_{0} \\
& =\sum_{I} W_{I} \exp \left(-K_{I} t\right)
\end{aligned}
$$

where $W_{I}$ means the initial fraction of substrates attached on the $I$-th residue.

Treatment 2: Kinetics Considering the Probability of Finding a Distribution is Derived from the Independent Propabilities for Each Residue

In this and succeeding sections, the cases in which substrates and catalytic groups are fixed on a chain during their reactions, will be treated. In these cases, the distribution of catalytic groups along the chain must be taken into account. In this section, the probability of finding a distribution is assumed to be determined by the probability for a given residue to carry a catalytic group, the probability being determined independently of the states of other residues. For simplicity, all kinds of the end effects are neglected here and the extention of the theory to the short chains will be discussed 


\section{Sisido}

later. The problem is to formulate the reaction course of total substrates, using the probability of a given substrate being surrounded by catalytic groups with a given distribution in the "reaction range" and the apparent rate constant determined by the distribution. It is put forword that only catalytic groups separated within $Z$ residues from a given substrate can act as a catalyst and this range is called the "reaction range."

Let us define $n_{i}(i=-Z, \ldots,-1,1, \ldots, Z)$ as the number (zero or one) of the catalytic groups attached on the $i$-th residue, then, the distribution of the catalytic groups in the reaction range can be represented by the $2 Z$ membered set of $n_{i}$ 's, $\boldsymbol{n}=\left(n_{-z}, \ldots, n_{z}\right)$. It should be mentioned here, that the numbering of the residues is made taking the position of the substrate as an origin. Putting $W(\boldsymbol{n})$ as the probability of a reaction range being in distribution $\boldsymbol{n}$, and $[R]_{0}$ as the initial concentration of the total substrates attached on chains, the initial concentration of the substrates $[R(n)]$, attached at the middle of the reaction range having the distribution $\boldsymbol{n}$ is given as

$$
[\mathrm{R}(\boldsymbol{n})]=W(\boldsymbol{n}) \cdot[\mathrm{R}]_{0}
$$

Using an apparent rate constant $K(\boldsymbol{n})$, for a substrate in the distribution $\boldsymbol{n}$, the fraction $y$, of unreacted substrates at time $t$, can be written in a similar form as eq 8

$$
y=\sum_{\boldsymbol{n}} W(\boldsymbol{n}) \exp (-K(\boldsymbol{n}) t)
$$

where the summation is made over all possible distributions. $W(\boldsymbol{n})$ and $K(\boldsymbol{n})$ can be written as

$$
\begin{aligned}
& W(\boldsymbol{n})=\prod_{\substack{i=-Z \\
\neq 0}}^{Z} p_{i}^{n_{i}}\left(1-p_{i}\right)^{\left(1-n_{i}\right)} \\
& K(\boldsymbol{n})=\sum_{\substack{i=-Z \\
\neq \neq}}^{Z} k_{i} \cdot n_{i}
\end{aligned}
$$

where $p_{i}$ means the probability for the $i$-th residue carrying a catalytic group as defined in treatment 1 , and it does not depend on the nature of the neighboring residues as stated above. $k_{i}$ represents the first-order rate constant of the reaction between a given substrate and a catalytic group on the $i$-th residue. Using eq 10,11 , and 12 , the total fraction of unreacted substrates is written as

$$
\begin{aligned}
y= & \sum_{-Z=0}^{1} \cdots \sum_{n_{-1}} \sum_{n_{1}} \cdots \sum_{n_{Z}} \prod_{\substack{i=-Z \\
\neq 0}}^{Z}\left\{p_{i}{ }^{n_{i}}\left(1-p_{i}\right)^{\left(1-n_{i}\right)}\right. \\
& \left.\times \exp \left(-k_{i} n_{i} t\right)\right\}
\end{aligned}
$$

The quantity enclosed by the parentheses, \{\} , depends only upon the nature of the $i$-th residue, therefore, eq 13 is rewritten in the form below.

$$
\begin{aligned}
y & =\prod_{\substack{i=-Z \\
\neq 0}}^{Z}\left\{\sum_{i=0}^{1} p_{i}{ }^{n_{i}}\left(1-p_{i}\right)^{\left(1-n_{i}\right)} \exp \left(-k_{i} n_{i} t\right)\right\} \\
& =\prod_{\substack{i=-Z \\
\neq \neq 0}}^{Z}\left\{1-p_{i}+p_{i} \exp \left(-k_{i} t\right)\right\}
\end{aligned}
$$

Eq 14 describes the whole reaction course required in this section. Usually, $p_{i}$ may be put as a constant value $p$, independently of $i$. Substituting zero for $t$ in eq 14, the initial fraction of unreacted substrates $y_{0}$, is found to be unity, and substituting an infinite value for $t$, the final fraction of unreacted substrates $y_{\infty}$, is obtained.

$$
y_{\infty}=\prod_{\substack{i=-Z \\ \neq \neq 0}}^{Z}\left(1-p_{i}\right)
$$

Eq 15 corresponds to the probability for a reaction range carrying no catalytic groups, as expected. The gradient in the first order plot is shown in eq 16 and the initial rate is in eq 17.

$$
\begin{gathered}
-\frac{\mathrm{d}(\ln y)}{\mathrm{d} t}=\sum_{i} \frac{p_{i} k_{i} \exp \left(-k_{i} t\right)}{\left\{1-p_{i}+p_{i} \exp \left(-k_{i} t\right)\right\}} \\
(\text { rate })_{0}=-\left(\frac{\mathrm{d} y}{\mathrm{~d} t}\right)_{t=0}=-\left(\frac{\mathrm{d} \ln y}{\mathrm{~d} t}\right)_{t=0}=\sum_{i} p_{i} k_{i}
\end{gathered}
$$

For a special case that all $k_{i}$ and $p_{i}$ are replaced by constant values $k$ and $p$ independently of $i$, eq 16 is rewritten in the following simple form:

$$
y=\{1-p+p \exp (-k t)\}^{2 Z}
$$

Treatment 3: Kinetics Considering That the Probability of Finding a Distribution is Derived from the Interdependent Probabilities

In this section, the polymer chain is treated in a manner that the probability for a reaction range in a given distribution of the catalytic 
groups along the chain is determined by the probabilities for each residue carrying a catalytic group, the probability depending upon the states of the neighboring residues. Strictly speaking, the case stated above includes two cases. The first case is that, for example, the distribution is determined by the copolymerization step, i.e., it is determined by the step-bystep mechanism subjected to the Markov process. ${ }^{8}$ In this case, the probability depends only upon the state of the preceding residue. The second case is realized by a rather rare process such that before the reaction begins the distribution is determined by the adsorption equilibrium and the reaction proceeds very fast compared with the rate of exchanging adsorption sites of the catalytic groups. In the second case the probability for a residue carrying a catalytic group depends upon not only the states of the neighboring residues but also those of all the residues in the chain. Therefore, these two cases must be treated separately. In this section the former case will be treated first and then the latter case will be considered.

In the former case, $n_{i}$ depends only upon $n_{i-1}$. Here the conditional probability $p\left(n_{i-1}\right.$, $\boldsymbol{n}_{i}$ ) is defined, as the probability for the $i$-th residue to carry $n_{i}$ catalytic groups providing that the $(i-1)$-th residue carries $n_{i-1}$ catalytic groups. Here the conditional probabilities are assumed to be represented by a universal function independent of $i$. Incidentally, if the chain is traced in an opposite direction, the probability for the $(i-1)$-th residue carrying $n_{i-1}$ catalytic groups depends only upon $n_{i}$. Therefore, the conditional probability $q\left(n_{j-1}, n_{j}\right)$ may also be defined, where the index $j$ is counted in an opposite direction to that used in the index $i$. If the end effect is ignored, these two probabilities coincide as

$$
p(m, n)=q(m, n) \quad m, n=0 \text { or } 1
$$

In this case, the probability of a reaction range being in the distribution $\boldsymbol{n}$ is

$$
\begin{array}{r}
W(n)=p\left(n_{0}, n_{1}\right) p\left(n_{1}, n_{2}\right) \cdots p\left(n_{Z-1}, n_{Z}\right) q\left(n_{0}, n_{-1}\right) \cdots \\
q\left(n_{-Z+1}, n_{-Z}\right)=p\left(n_{0}, n_{1}\right) \cdots p\left(n_{Z-1}, n_{Z}\right) p\left(n_{0}, n_{-1}\right) \cdots \\
p\left(n_{-Z+1}, n_{-Z}\right)
\end{array}
$$

Using eq 10 and 20

$$
y=\left\{\sum_{n_{1}=0}^{1} \cdots \sum_{n_{Z}} \prod_{i=1}^{Z} p\left(n_{i-1}, n_{i}\right) \exp \left(-k_{i} n_{i} t\right)\right\}^{2}
$$

where $k_{i}=k_{-i}$ is assumed. This assumption is absolutely correct in non-directional polymers (e.g., vinyl polymers) and approximately correct in directional ones (e.g., $\operatorname{poly}(\alpha$-amino acid)s). Eq 21 becomes simpler using matrices $\mathbf{A}_{i}$ 's, each of which is defind as

$$
\mathbf{A}_{i}=\left[\begin{array}{ll}
p(0,0) & p(0,1) \exp \left(-k_{i} t\right) \\
p(1,0) & p(1,1) \exp \left(-k_{i} t\right)
\end{array}\right]
$$

Using the elements of $\mathbf{A}_{i}$ 's, eq 21 is rewritten as

$$
\begin{aligned}
y & =\left[\sum_{m_{1}=1}^{2} \cdots \sum_{m_{Z}}\left\{\mathbf{A}_{\mathbf{1}}\right\}_{m_{0}},{ }_{m_{1}} \cdots\left\{\mathbf{A}_{Z}\right\}_{m Z-1}, m_{Z}\right]^{2} \\
& =\left[\sum_{m_{Z}}\left\{\mathbf{A}_{1} \cdots \mathbf{A}_{Z}\right\}_{m_{0}}, m_{Z}\right]^{2}
\end{aligned}
$$

Eq 23 describes the total reaction course of the polymer reaction required. $m_{0}\left(=n_{0}+1\right)$ must be determined according to experimental conditions. In general, when the block character in the distribution of catalytic groups is large $(p(1,1) \approx$ 1), this selection becomes important.

In a special case where all $k_{i}$ 's are equivalent to $k$, eq 23 can be reduced to a very simple form as below. When $p(0,0)$ is not very different from $p(1,0)$, the first row of matrix $\left(\mathbf{A}_{1}\right.$ $\cdots A_{Z}$ ) becomes almost equal to the second row for sufficiently large $Z$. Therefore, in these cases the cyclic condition $\left(m_{0}=m_{Z}\right)$ can be used in eq 23 .

$$
y=\left[\operatorname{Trace}\left\{\mathbf{A}_{1} \cdots \mathbf{A}_{z}\right\}\right]^{2}
$$

The equivalence of $k_{i}$ 's leads to the equivalence of all $\mathbf{A}_{i}$ 's, and therefore, taking all $\mathbf{A}_{i}{ }^{\prime}$ 's as $\mathbf{A}$.

$$
y=\left[\text { Trace }\left\{\mathbf{A}^{Z}\right\}\right]^{2}=\left(\lambda_{1}{ }^{Z}+\lambda_{2}{ }^{Z}\right)^{2}
$$

where, $\lambda_{1}$ and $\lambda_{2}$ are the eigenvalues of matrix A. Taking the largest eigenvalue to be $\lambda_{\max }$, eq 25 is written in an approximate form for sufficiently large $Z$.

$$
y=\lambda_{\max }^{2 Z}
$$

Substituting an infinite value for $t$, the eigenvalue of $\mathbf{A}$ is calculated to be zero or $p(0,0)$. This gives $y_{\infty}=\{p(0,0)\}^{2 Z}$ which again corresponds to the probability that no catalytic group is attached on any residue in the reaction range. On the other hand, when zero is substituted for $t$, two eigenvalues become 1 
and $p(0,0)-p(1,0)$. These values lead to the correct value of $y_{0}(=1)$ for large $Z$. When $p(0,0)=p(1,0)$, eq 25 coincides with eq 18 , showing the consistency of these equations.

The second case mentioned above should now be considered. In this the distribution of the catalytic groups should be treated by the Ising theory of a one-dimensional cooperative system. ${ }^{9}$ Using this theory, the conditional probability $p\left(n_{i-1}, n_{i}\right)$ can be obtained by the following procedure. Matrix $\mathbf{G}$ is defined as $^{9}$

$$
\mathbf{G}=\left[\begin{array}{ll}
\exp (-\varepsilon(0,0) / \boldsymbol{k} T) & \exp (-\varepsilon(0,1) / \boldsymbol{k} T) \\
\exp (-\varepsilon(1,0) / \boldsymbol{k} T) & \exp (-\varepsilon(1,1) / \boldsymbol{k} T)
\end{array}\right]
$$

where, $\varepsilon\left(n_{i-1}, n_{i}\right)$ is an energy assigned to the $i$-th residue when the $(i-1)$-th residue has $n_{i-1}$ catalytic groups and the $i$-th residue has $n_{i}$. Taking $\mu$ as the largest eigenvalue of $\mathbf{G}$, the conditional probability required is given as ${ }^{9}$

$$
p\left(n_{i-1}, n_{i}\right)=\left\{\exp \left(-\varepsilon\left(n_{i-1}, n_{i}\right) / k T\right) / \mu\right\}\left(v_{m_{i}} / v_{m_{i-1}}\right)
$$

where, $v_{m_{i}}$ means the element of eigenvector $v$ corresponding to the eigenvalue $\mu$, and $m_{i}=$ $n_{i}+1$.

$$
\mathbf{G} \boldsymbol{v}=\mu \boldsymbol{v}, \quad \boldsymbol{v}^{+}=\left(v_{1}, v_{2}\right)
$$

Using the conditional probability represented by eq 28 , the reaction course can be calculated as described before.

\section{DISCUSSION}

\section{Remarks on the Application to the Short Chains}

The first treatment, in which no distribution was taken into consideration, can in principle be applied to chains having any degree of polymerization. Recently, eq 4 was applied to the initiation step of the polymerization of amino acid NCA by poly(vinylpyridine)s having various chain lengths. ${ }^{10}$ On the other hand, the kinetic theories in treatments 2 and 3 were developed assuming the infinite chain length. Therefore, when the approximation of infinite chain length is not acceptable, i.e., number of residues in the reaction range $2 Z$ is comparable with the degree of polymerization $N$, the differences in the apparent rate constants, caused by the differences in the positions of substrates on a chain, must be considered. In this case, the substrate must be discriminated by the position, $I$, on the chain, counting the residues from an end of the chain as defined in treatment 1 . If $I$ or $(N-I)$ is smaller than $Z$, the lower or the upper limit of the product in eq 14 must be taken off respectively. Then the obtained fraction of unreacted substrates attached to the $I$-th residue $y_{I}$, must be averaged to obtain the total fraction $y$

$$
y=\sum_{I} y_{I} / N
$$

where uniform distribution of the substrates is assumed at the beginning of the reaction. In treatment 3 , a further precaution must be taken with the use of cyclic condition, as stated previously.

\section{Dependence of the Rate Constant upon the Re- lative Separation between a Substrate and a Catalytic Group}

In the kinetic treatments developed in this paper, two kinds of parameters were used, One is related to the probability of the existence of a catalytic group on a given residue and the other is the rate constant depending upon the separation along the chain. Among these parameters, the probabilities can usually be considered to be independent of the position of a residue. On the other hand, the dependence of $k_{i}$ upon $i$ is important and has been studied by several workers. For example, the relative ease of the lactone formation from $\omega$-oxyacids having various chain lengths from 5 to 24 was studied by Stoll, et al. ${ }^{11}$ Their treatment of the experimental data was recently modified by Morawetz, et al. ${ }^{12}$ Also for short polymethylene chains, the statistical estimation of the rate constants using $G-T$ rotational isomer model for each $\mathrm{C}-\mathrm{C}$ bond was recently performed by the author. ${ }^{13}$ Concerning other chains, the dependence of the intramolecular rate constant upon the separation between two functional groups along a chain was investigated with several poly $(N$-alkylamino acid $) s^{14-16}$ On the other hand, for longer chains, Morawetz, et al., 5 proposed the following equation for polyacrylamide chains,

$$
k_{i}=k|i|^{-a}(10 \leq|i| \leq 500), \quad k_{i}=0 \text { (otherwise) }
$$

where $k$ is a constant coefficient and $a$ is the 
ring-closure exponent which should be 1.5 for Gaussian chains. They stated, however, that it is better to take $a=2.0$, to match their experimental results.

\section{Examples}

Morawetz, et al., ${ }^{5,6}$ studied the intramolecular hydrolysis reaction and performed computer simulation of the reaction course assuming a random distribution of catalytic groups. They used terpolymers including monomers I and II as a substrate and a catalytic group respectively and acrylamide as an inactive residue. They
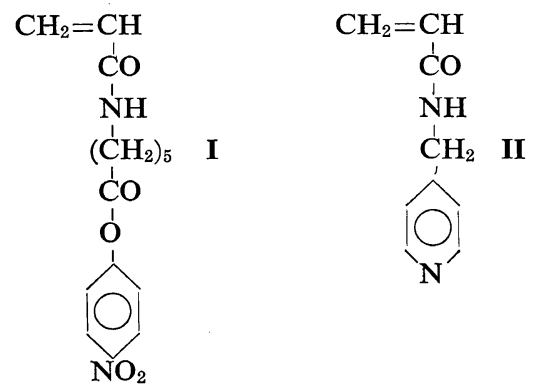

measured the intramolecular reaction rate between the side groups on the chain, that is, the hydrolysis of $p$-nitrophenyl ester group by the pyridine ring. In this section, similar calculations were made by the analytical equations developed above.

\section{Application of the Treatment 2}

In this case, the probability of finding a distribution of catalytic groups is considered to be derived from the independent probabilities for each residue. According to Morawetz, et al., ${ }^{5,6}$ the parameters for the calculation were given as

$$
\begin{gathered}
Z=500, p_{i}=p=\omega=0.0033,0.012, \text { and } 0.048 \\
k_{i}=\alpha k|i|^{-a} \quad(10 \leq|i| \leq 500), k_{i}=0 \text { (otherwise) } \\
\quad \alpha=0.2, \quad a=2.0 \text { or } 1.5
\end{gathered}
$$

where $\alpha$ means the fraction of pyridine residue in its basic form. Since the interchange between active and inactive forms is considered to occur very frequently during the reaction, the active pyridyl groups can be regarded as distributed among the whole pyridyl groups uniformly. Therefore, each rate constant should be multiplied by the fraction of active form $\alpha$, as shown above. Degree of polymerization of their polymer was 4900 , so any kind of end effect can be ignored. The coefficient $k$ was determined from the observed initial slope of the reaction course at $p=0.048$ using eq 17 .

The results are shown in Figures 1 and 2 .

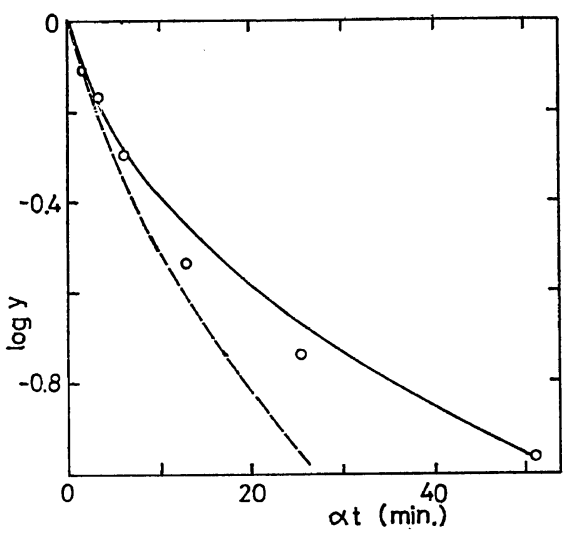

Figure 1. First-order plot of the hydrolysis of acrylamide terpolymer $(\omega=0.012): \bigcirc$, experimental points; ${ }^{6}-$, calculated curve using eq 14 , at $a=2.0 ;-\cdots$, at $a=1.5$.

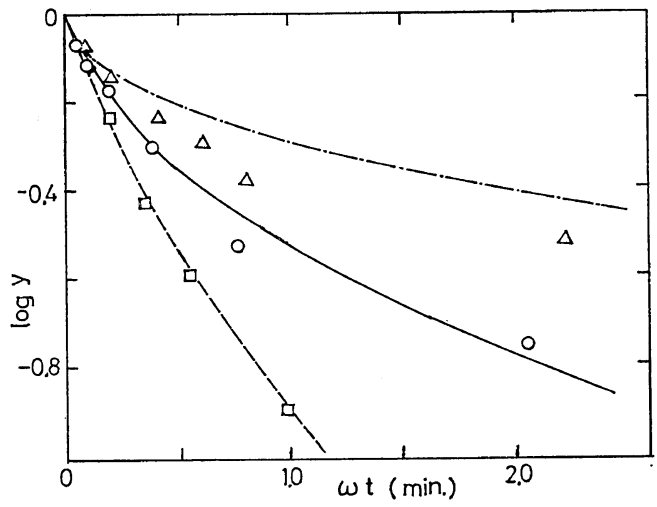

Figure 2. Calculated curves for the first-order plot of the hydrolysis of acrylamide terpolymers using eq 14: $-\cdot-\triangle-\cdot-, \omega=0.0033 ;-\bigcirc-$ $\omega=0.012 ; \quad-\square--, \omega=0.048 ; \quad \triangle, \bigcirc, \square$, experimental points. ${ }^{6}$

The effect of varying ring-closure exponent $a$, is shown in Figure 1. It is shown that the calculated curve taking $a=2.0$ is in better agreement with the experimental points than the curve obtained taking $a=1.5$, as already stated by Morawetz, et $a l^{5,6}$ The effect of varying the mole fraction of catalytic monomer units 


\section{Sisido}

$(\omega=p)$ is shown in Figure 2, in which the calculated curves were obtained taking $a=2.0$ and using the same coefficient $k$ as used in Figure 1. In the case of $p=0.0033$, the agreement with experimental values is rather poor. However, in the other two cases, the calculated curves and the experimental points are in close agreement.

\section{Application of the Treatment 3}

Here, the same experimental results will be treated assuming that the probability of finding a catalytic group on a residue depends on the state of the neighboring residue. The conditional probabilities $p(0,1)=p_{0}, p(1,1)=p_{1}$, were varied to satisfy the condition that the total fraction $\omega$, of the catalytic monomers in the terpolymer be fixed to a constant value, 0.048 .

$$
\omega\left(1-p_{1}\right)=(1-\omega) p_{0}, \quad \omega=0.048
$$

As $p_{1}$ approaches zero, the distribution becomes alternative and as $p_{1}$ approaches unity, the block character of the distribution becomes marked. Calculations were made by eq 23 taking $n_{0}$ to be zero. Also in this case, the coefficient of the rate constant $k$ had to be determined from the initial rate. Since the differentiation of eq 23 with time is complicated, $k \alpha$ was determined as a ratio of the initial rate actually observed, to that determined by the numerical differenti-

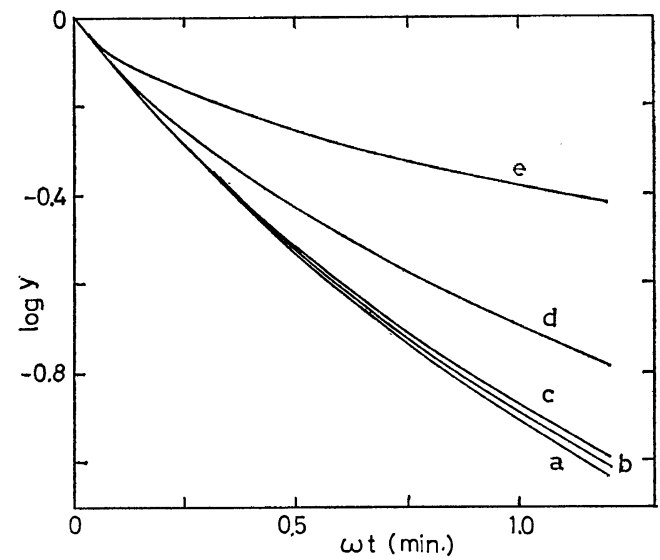

Figure 3. Calculated curves for the first-order plot of the hydrolysis of acrylamide terpolymer using eq 23: a, $p_{0}=0.0504, p_{1}=0.0 ; \quad \mathrm{b}, p_{0}=p_{1}=$ $0.048 ; \quad \mathrm{c}, p_{0}=0.0454, p_{1}=0.1 ; \mathrm{d}, p_{0}=0.0252, p_{1}=$ $0.5 ; \quad$ e, $p_{0}=0.005, p_{1}=0.9$. ation of eq 23 taking $t=0$ and $k \alpha=1$. The validity of this procedure is supported by considering that the value of the first derivative of $y$ at $t=0$ is equal to the coefficient of the firstorder term of $t$ in the expanded form of $y$, and the coefficient is also the first-order with reference to $k \alpha$ as seen from the representation of $y$ in eq 23. The results are shown in Figure 3. It was found that for the polymers having such a low fraction $\omega$ of catalytic groups as 0.048 , the changes in the reaction profiles induced by the change of the probabilities were not so large except in these cases where the block character was extremely large.

In conclusion, the kinetic theories for the reactions proceeding on a polymer chain were developed. In these treatments, the parameters $k_{i}$ 's were used. It is very important to clarify the dependence of $k_{i}$ upon $i$ both experimentally and theoretically for a better understanding of the polymer reactions.

Acknowledgement. The author is indebted to Prof. T. Higashimura and Dr. Y. Imanishi for helpful discussions. All computations were carried out at the Data Processing Center at Kyoto University.

\section{REFERENCES}

1. H. Morawetz and P. E. Zimmering, J. Phys. Chem., 58, 753 (1954).

2. E.W. Westhead, Jr. and H. Morawetz, J. Amer. Chem. Soc., 80, 237 (1958).

3. E. Gaetjens and H. Morawatz, J. Amer. Chem. Soc., 83, 1738 (1961).

4. G. Smets and A. M. Hesbain, J. Polym. Sci., 40, 217 (1959).

5. H. Morawetz, Account Chem. Res., 3, 354 (1970).

6. N. Goodman and H. Morawetz, J. Polym. Sci., Part C, 31, 177 (1970).

7. L. Michaelis and M. L. Menten, Biochem. Z., 49, 333 (1913).

8. G. E. Ham, ed., “Copolymerization," Interscience Publishers, Inc., New York, N.Y., 1964, Chapter 1.

9. T. M. Birshtein and O. B. Ptitsyn, "Conformations of Macromolecules," S.N. Timasheff and M.J. Timasheff, Translators, Interscience Publishers, Inc., New York, N. Y., 1966, Chapter 5.

10. S. Nagaoka, K. Suzuoki, Y. Imanishi, and T. Higashimura, in preparation.

11. M. Stoll and A. Rouvé, Helv. Chim. Acta, 18, 


\section{Kinetic Theory for Polymer Reactions}

1087 (1935).

12. H. Morawetz and N. Goodman, Macromolecules, 3, 699 (1970).

13. M. Sisido, Macromolecules, 4, 737 (1971).

14. D.G.H. Ballard and C.H. Bamford, Proc. Roy.
Soc., Ser. A, 236, 384 (1956).

15. M. Sisido, Y. Imanishi, and S. Okamura, Biopolymers, 7, 937 (1969).

16. M. Sisido, Y. Imanishi, aud S. Okamura, Polymer J., 1, 198 (1970). 